\title{
Embedded Sensing: Making the best of 3D printed sensors
}

\author{
Alexander Dijkshoorn, Patrick Werkman, Marcel Welleweerd, \\ Gerjan Wolterink, John Delamare, Remco Sanders, Gijs J.M. Krijnen \\ Robotics and Mechatronics group, MIRA institute, University of Twente, Enschede, The Netherlands \\ Corresponding author: gijs.krijnen@utwente.nl
}

\begin{abstract}
Current Additive Manufacturing allows for the implementation of electrically interrogated 3D printed sensors. In this contribution various technologies, sensing principles and applications are discussed. We will give both an overview of some of the sensors presented in literature as well as some of our own 3D printed sensors work.
\end{abstract}

Key words: 3D printing, embedded sensors, force sensing, strain sensing, EMG sensing.

\section{Introduction}

Additive Manufacturing (AM), more colloquial known as $3 \mathrm{D}$ printing, is a digital fabrication technology in which parts are built layer-by-layer using one method out of a variety of different methods to deposit and solidify specific materials. Meanwhile the technology has been around for more than 3 decades but recently the number of materials that can be printed, the resolution and speed with which this can be done and the ability to combine more than one material in multimaterial prints have been improved significantly, while on the other hand the costs of printing and materials have steadily declined.

AM has been used traditionally to make nonfunctional structures for rapid prototyping purposes. However, recent developments in multimaterial 3D printing have started to trigger research into printing of functional structures [1]. For customised structures that already are made by $A M$, e.g. robotics and prosthetics, embedding 3D printed sensors seems a promising next step to increase functionality. The development of this technology will allow application of sensors, independent of commercial-of-the-shelve (COTS) components, since customisation in performance, packaging and interfacing, as well as integration in/with complex shapes, will lead to unprecedented possibilities. Where the performance of such sensors will have the burden of proving their viability relative to other wellestablished sensor technologies like MEMS and precision engineering [2], it is clear that developments in e.g. soft-robotics, prosthetics, orthotics and other 'soft-material' based developments require sensors largely different from current COTS sensors. At the same time fully integrated manufacturing may come at reduced costs since no assembly will be required.

Clearly, embedded sensing and actuation cannot do without proper electronic underpinning. However, despite the wide interest in (3D) printed electronics, we do not consider such integration in this paper since we believe that such developments will become only available much further into the future. Nevertheless the combination of electronics components with 3D printed structures through manual or (semi-)automated assembly has already been demonstrated [3] and will allow tight integration with embedded transduction.

Current technology for $3 D$ printed sensors Currently there are three methods to combine dielectric with conductive parts in order to make sensors and actuators:

A) hybrid approaches, i.e. combining printed parts with non AM fabricated structures, e.g. regular wiring, printed circuit boards or entire sensors,

B) conductor infusion, i.e. printing channels in otherwise non-conductive materials by arbitrary $A M$ methods with subsequent infusion of conductive inks [4], and

C) by multi-material printing, i.e. combining the 
use of conductive and non-conductive filaments [1], predominantly by Fused Deposition Modeling (FDM).

Method A) allows for a straightforward combination of parts, largely comparable to classical assembly. The disadvantage is that the potential of AM is only materialised in a very limited way. The advantage of $\mathrm{C}$ ) is the promise of straightforward integration, its disadvantage the anisotropic conduction. Method B) does not have this disadvantage but homogeneously filling channels with conductive inks is challenging, especially when filling needs to be obtained by pressure driven flows and through a diverse channel network. With respect to electrical interfacing both methods $\mathrm{B}$ ) and C) can show considerable variations in contact resistances, subject to mechanical loading, temperature and humidity changes, etc.

\section{Hybrid approaches}

Hybrid approaches can offer the best of 'both worlds': freeform fabrication and proven technology for specific parts, i.e. the read-out of a sensor. Although it presents limitations relative to multi-material printing it generally provides easy approaches and solutions for functional integration.

\section{Whisker inspired tactile sensor}

We recently investigated possibilities to 3D print biomimetic whisker sensors for tactile purposes [5]. In these studies we set-out for a hybrid integration approach: the whisker structure and its suspension are 3D printed whereas the readout consists of a capacitive measurement of a co-planar capacitance, affected by a dielectric driven into the electric field of the capacitance. The implementation contains a PCB providing the required electrode structures. A schematic of the sensor is shown in Fig. 1.

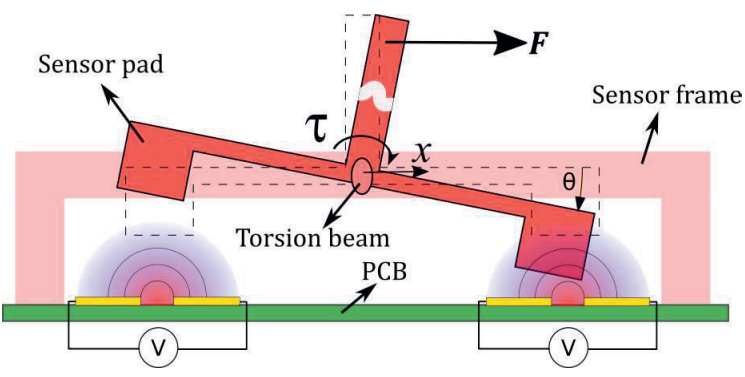

Fig. 1: Coplanar capacitive sensing using interdigitated fingers with dielectric loading.

Examples of the design and printed parts are shown in Fig. 2. The rotation is enabled by a torsion beam with elliptical cross-section, giving more compliance in the horizontal than in the vertical direction. An arbitrary force acting at an arbitrary position on the whisker will result in both a lateral force and a torque with subsequent rotation angle and lateral shift.
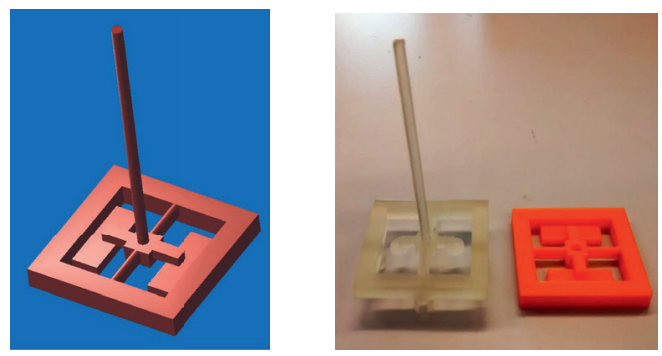

Fig. 2: Left: $3 D$ CAD-design of whisker sensor in OpenScad [6]. Right: Printed whisker sensor (transparent, Veroclear) and base (red, PLA).

Fig. 3 shows how the suspension behaves under mechanical loading. Overall the observed rotation angle is well in line with the predicted rotation angle. However, there is a significant difference between loading curves (open symbols) and unloading curves (solid symbols), reminiscent of mechanical hysteresis.

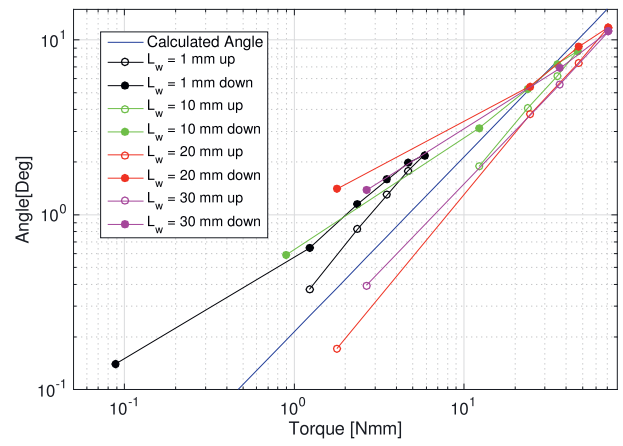

Fig. 3: Rotation angle versus torque

Although sensor structure and read-out were not integrated in this work we investigated the effect of the dielectric in the electrical field of the co-planar capacitor. Fig. 4 shows the results of these measurements where we used an HP4284A LCR meter to determine the capacitance and a linear translator to displace the dielectric. Two curves measured at $1 \mathrm{MHz}$ (red triangles and blue dots) clearly constitute a hysteresis curve. Also some odd behaviour can be seen at separation distances below $0.2 \mathrm{~mm}$ which we attribute to mechanical deformation of the dielectric pad and/or the PCB when mechanically loaded. We have shifted these curves $-0.2 \mathrm{~mm}$ to better reflect and compare them with 
the curve measured at $500 \mathrm{kHz}$ and the two calculated curves.

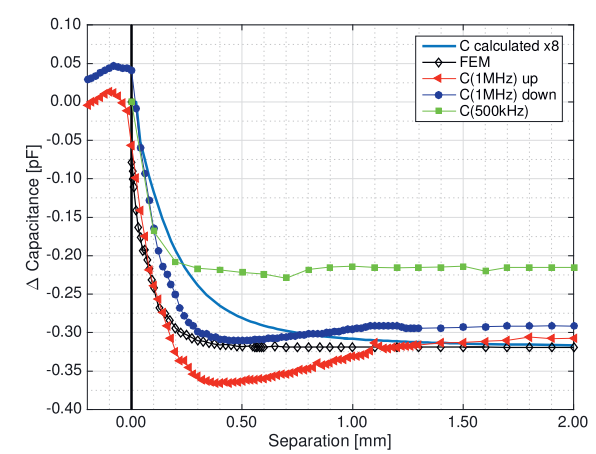

Fig. 4: Coplanar capacitive sensing: capacitance vs distance of dielectric load.

In the measurements we obtained a maximum change in capacitance of about $0.35 \mathrm{pF}$ relative to a base value of about $2.1 \mathrm{pF}$, i.e. $\triangle C / C \approx$ 0.17 . Comparable values are found in the FEM calculations but are under-estimated by a factor of about 8 using conformal mapping calculations.

It is important to observe that both the mechanical as well as the capacitive measurements clearly show hysteresis behaviour, a feature observed with virtually all our 3D printed sensors consisting of plastics.

\section{Infused conductors}

Making electrical conductors by filling channels has the benefit of having freedom of choice of the 3D print process as long as it a) allows for the use of soluble support material to make channels and $b$ ) is sufficiently impermeable to the fluids later to be infused into the channels. In principle wetting surfaces could allow for filling by capillary forces but in practice the particles laden fluids and glues have viscosities too high to obtain significant filling before drying. Further complications can stem from the need to fill complex networks and the need for a fine balance between the time to fill the channels and the time needed for solidification of the infused material ${ }^{1}$.

\section{DOF force sensor}

In a recent research project we have investigated the possibilities to make a 3DOF force sensor by having an inclusion sitting on a membrane containing a multiple of strain-gauges and capacitive sensors, see Fig. 5. The design freedom in AM allows to make channels that follow the lines of

\footnotetext{
${ }^{1}$ In principle fluidic conductors could be used as well but these tent to be expensive and require impermeable channelwalls.
}

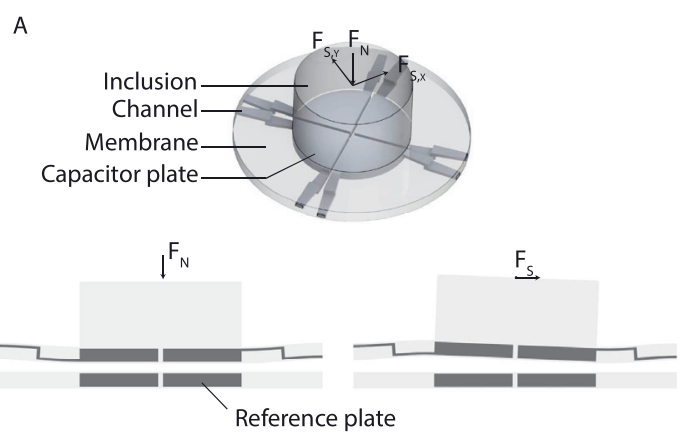

Fig. 5: Design of a 3DOF force sensor with infused conductors.

maximum strain through the membrane, hence switching from top to bottom in the membrane in the current design.

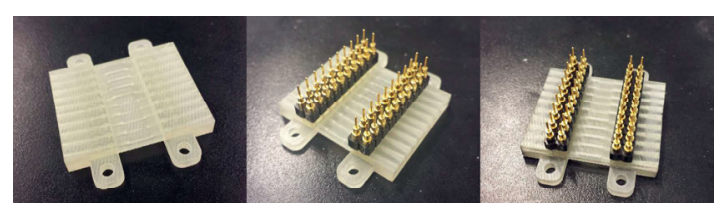

Fig. 6: Assembly of a strain gauge sensor with straight channels. From left to right: The chip as received from Shapeways, the chip after insertion of headers and cleaning, the chip after infusion with graphite paint.

To investigate the quality of the infused conductors and study the mechanical and strain-gauge performance a series of straight and cross-over channels were made, see Fig. 6. Special care was taken to design appropriately shaped room for the header-pins which otherwise may be subject to large variations in contact resistance.

Mechanical loading tests were carried out on a test rig consisting of a voice coil actuator connected to a load cell via a rod sliding over an air bearing. Forces are applied by means of a plunger, connected to the load cell whereas the resulting displacements are measured simultaneously. The system is set to a certain force after which the resistance of the ten channels, the load cell and the displacement sensor are read out.

Mechanical response of the strain-gauge to repetitive loading is shown in Fig. 7 . Clearly the mechanical response shows a) nonlinearity, b) hysteresis and c) creep or drift as evidenced by the increasing deflection at 0 load. The effects seem to be stronger when the structures are loaded from the bottom (left) than from the top (right). A nonlinear beam model, taking into account beam-stiffening and based on [7], shows a reasonable correspondence in shape, exclud- 

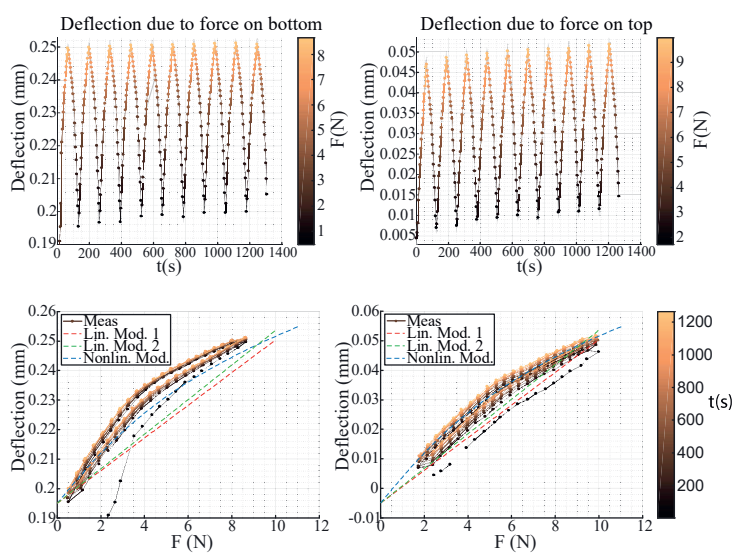

Fig. 7: Repetitive mechanical loading of the straingauges. Deflection versus time with colour-coding for the load (top) and deflection versus load with colourcoded time (bottom).

ing the hysteresis. However, the displacements at which beam stiffening seems to occur are relatively low suggesting that other effects than beam-stiffening (only) may be at play.
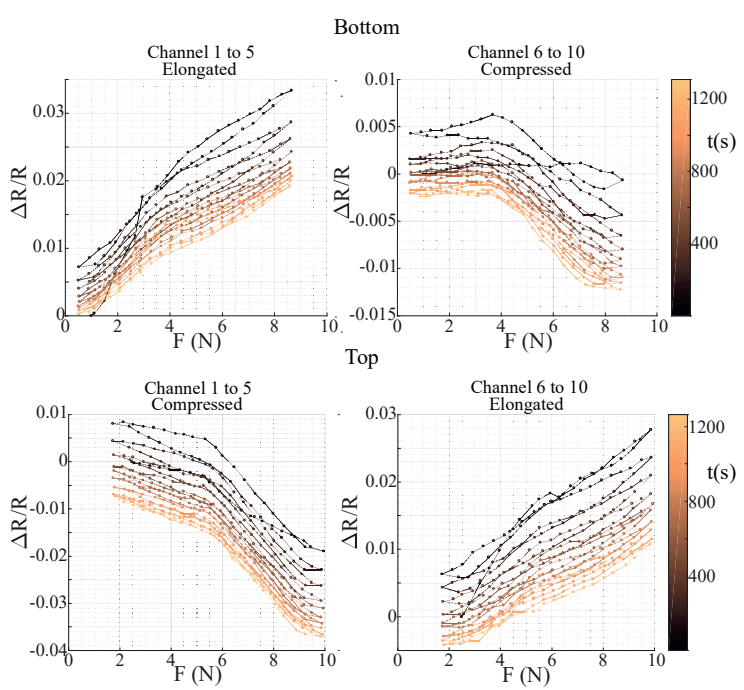

Fig. 8: Repetitive mechanical loading of the straingauges. Relative resistance change versus load with colour-coded time.

When looking at the electrical response the mechanical response seems to be largely reflected though the creep seems to be more significant.

\section{Co-printed conductors}

There is a steady increase in availability of multimaterial printers [8]. Especially multi-nozzle FDM printers may process up to 5 materials [9] from an ever increasing range of specialty filaments. Some printers directly target co-printed conductive materials for printed electronics and embedded sensing [10].

\section{Piezo-resistive sensing}

Here we describe some preliminary results of strain-sensors consisting of co-printed dielectric beams and conductors.

\section{Conductive Filament \& Properties}

Various conductive materials are commercially available for FDM printing. In this research we used Proto-pasta filament because of its high resisitivity (yielding an easy measurement setup). The filament consists of PLA with carbon black filling as conductive agent [11]. Carbon black (CB) is a term for various types of ultrafine paracrystalline carbon particles (10-400 $\mathrm{nm}$ diameter) [12]. CB has a high heat resistance, high chemical resistance, a low mass density, low thermal expansion and provides electrical conductivity which makes it suited as filler in polymers. Several mechanisms may be involved in the conductivity of CB filled conducting polymers, including quantum mechanical tunneling, thermal expansion and mechanical interactions according to percolation theory [13].

Proofs of concept for the application of the material in strain sensing research have, amongst others, focused on silicone [14] and PolyLactic Acid (PLA) with CB [1]. Disadvantages of CB doped polymers are their high electrical resistance (making it only suited for low current applications) and their large creep deformation [15].

Proto-pasta filament is compatible with any PLA capable printer. The mechanical properties are comparable to undoped PLA, while the layer adhesion is worse [11]. Due to anisotropy, the tensile strength and elastic properties of the part will vary depending on the built orientation [16]. The material shows increased brittleness relative to undoped PLA.

\section{Experiments \& Results}

Experiments where done to determine both electrical and mechanical properties. Using 4 wire measurements to eliminate contact resistances $(30 \Omega)$, the gauge-resistances were determined by means of a source-meter (Keithley 2440). Tests of the conductive properties showed that Proto-pasta is an ohmic material with a strong temperature dependent resistivity. The resistivity could be determined for different printing orientations see Fig. 9. The resistivity of the material is determined, see table 1 .

To determine the piezoresistive effect a tensile test was performed with a universal tensile tester 


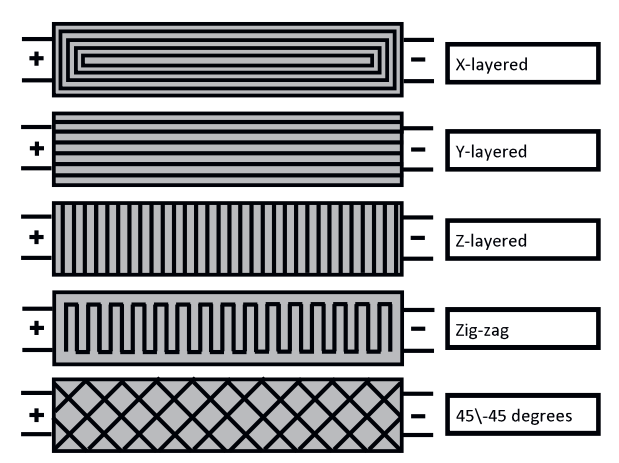

Fig. 9: Different beam printing orientations for the resistivity experiments

Table 1: Resistivity for different printing orientations and infill patterns

\begin{tabular}{l|c} 
Orientation & Resistivity $/ \Omega \mathrm{cm}$ \\
\hline X-layered & 10.90 \\
Y-layered & 9.00 \\
Z-layered & 9.11 \\
Zig-zag & 26.10 \\
45 \-45 degrees & 11.50
\end{tabular}

(Zwick, Model Z1.0) in combination with a 4 wire measurement. The change in resistance is plotted against the strain (figure 10). The correlation appears to be linear with exception of the interval $\left[0,3 \cdot 10^{-3}\right]$ (additional tests confirmed the linear behaviour for higher strains). The same nonlinear behaviour at small $\varepsilon$ was found in literature for straining polymers with carbon nano-particles [17]. A possible explanation, as given in [18], is the competition between structural changes due to the elongation and reorganisation of the conducting network .

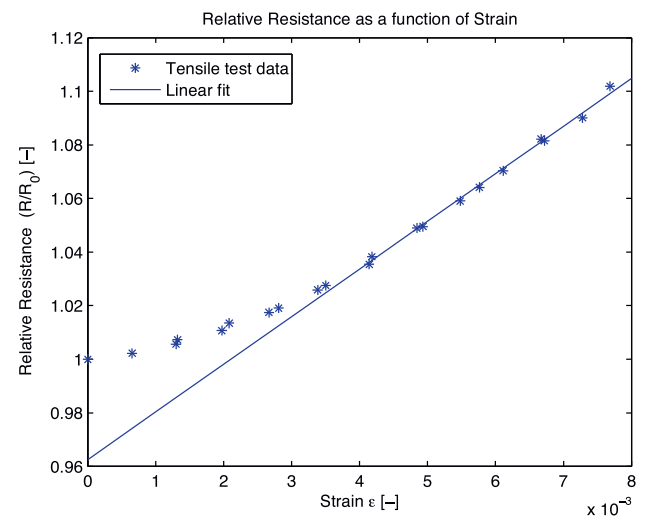

Fig. 10: Resistance vs strain of tensile test

From the obtained results a gauge factor can be derived, given by the ratio between relative change of resistance to mechanical strain. It is a combination of geometric terms and the piezoresistive effect. From the slope of the linear region in figure 10, the gauge factor of the material,
$K=\frac{\Delta R / R_{0}}{\Delta \varepsilon}$, can be determined to be approximately 17.8 . This gauge factor is high enough to provide usable results in various applications.

Finally, as proof on concept, a planar and an integrated complex strain sensor were printed (figure 11). Resistances were measured and the functioning of the strain sensors were demonstrated qualitatively. From these experiments it can be concluded that Proto-pasta is suited for printing strain sensors. With Additive manufacturing integrated strain sensors new advanced structures like in figure 11 are feasible.

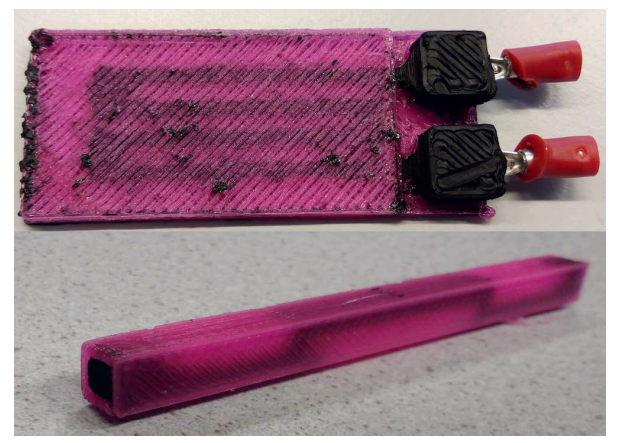

Fig. 11: 3D Printed integrated strain sensors

\section{FDM printed elastic SEMG sensors}

One of the exciting applications of embedded sensing is in the realm of soft robotics and medical applications such as prosthesis and orthoses. These applications have in common that they contain soft structures which are difficult to sensorize traditionally. However, recent developments of both materials and printers have opened new possibilities for flexible prints, despite FDM being a relatively poor proposition for flexible materials compared to e.g. moulding and poly-jetting. In this research we have used a FlashForge Creator Pro [19] printer, modified with a direct drive Flexion extruder [20], essential for printing flexible materials. Both the dielectric (NinjaFlex [21]) and conductive (Blackmagic [22]) filaments are thermoplastic polyurethane (TPU) based and highly stretchable.

In this study we are aiming for highly deformable surface electro myo-graphy (sEMG) sensors with the potential to make larger arrays, customised fits, to integrate shielding, etc. An impression of such structures can be seen in Fig. 12.

Utilising such structures we tested the possibility to capture muscle activity induced signals. 3D printed sensors were placed above the biceps brachii of a subject according to SENIAM recommendations [23] and we used a TMSi Refa am- 


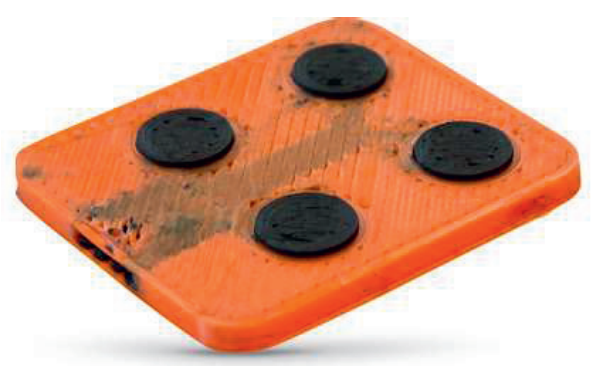

Fig. 12: Photograph of $3 D$ printed SEMG electrodes printed using a combination of conductive and dielectric TPU.

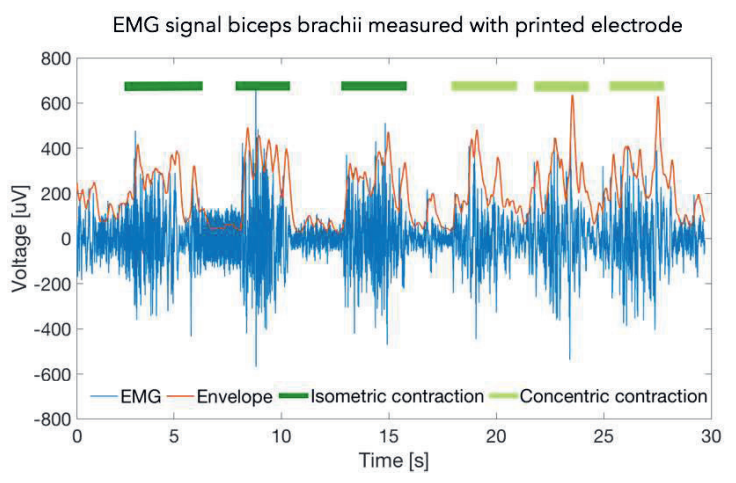

Fig. 13: Electrical signals registered using a $3 D$ printed flexible sEMG sensor. Blue: raw signal after 5 $\mathrm{Hz}$ 2nd order high-pass filtering and $50 \mathrm{~Hz}$ notch filtering. Red: envelope of the signal. Green bars indicate muscle activity.

plifier to read, amplify and digitise the sEMG signals. Subsequently these were filtered $(5 \mathrm{~Hz}$ 2nd order high pass filter and $50 \mathrm{~Hz}$ notch fitler) using MatLab. First results of such measurements are shown in Fig. 13 and indicate high correlation between electrode signal and muscle activity. In comparison with regular $\mathrm{AgCl}$ electrodes, Fig. 14, we see that the printed sEMG signals are a) in the same range, b) have more noise and c) have lower energy in the higher frequencies relative to the $\mathrm{AgCl}$ sensors (not shown here).

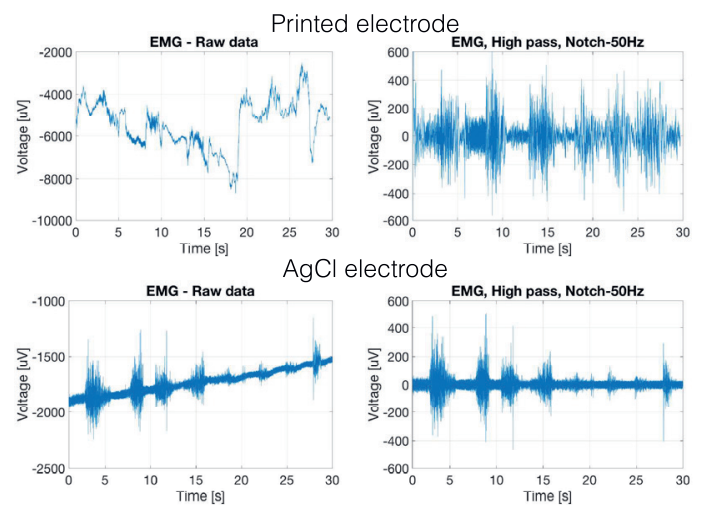

Fig. 14: Comparison of sEMG signals acquired with printed and regular $\mathrm{AgCl}$ electrodes

\section{Discussion}

In the examples given in this paper we have seen many non-idealities originating from mechanical sources, electrical sources or both. As far as plastics are involved this may be something we may expect. It would be straightforward to dismiss 3D printed sensors for this reason. However, this would also mean that we miss out on the large potential of 3D printed sensors, while adhering to the ideal engineering world, mainly consisting of linear systems. Nature tells us a different story; the entire sensing system of the animal kingdom is based on nonlinear sensing as implied by the properties of neural systems. At the same time it is easy to see these systems perform extremely well. Hence, rather than abandoning 3D printed sensors it becomes extremely interesting to think of systems which are resilient to the nonlinearities of sensors. E.g. by smart signal processing (for example the effects of creep and drift can be alleviated by high-pass filtering). Or by measurement and control strategies that are aware of nonlinearities. With the advent of 3D printed sensors it will become increasingly easy to deploy many sensor(array)s. Nature forms a living example of what that may bring.

\section{Conclusion}

We have discussed various methods to make embedded sensors by 3D printing and have given examples of these methods by sensors we have developed in our lab; piezo-resistive sensors (strain-gauges), capacitive read-out and bio-electric sensing. A more or less common theme of these sensors seems to be their nonidealities in the form of non-linearities, creep and hysteresis. Since the development of 3D printed sensors opens a potential too large to be disregarded, our future research will be dedicated to innovative approaches to make ample use of these sensors.

\section{References}

[1] S. J. Leigh et al., 'A Simple, Low-Cost Conductive Composite Material for 3D Printing of Electronic Sensors,' PLOS ONE, 7(2012)(11):pp. 1, doi:10.1371/journal.pone.0049365.

[2] G. J. Krijnen et al., 'Recent Developments in Bio-Inspired Sensors Fabricated by Additive Manufacturing Technologies,' Advances in Science and Technology, 100(2016):pp. 197, doi:10.4028/www.scientific.net/AST.100.197.

[3] C. Shemelya et al., 'Multi-functional 3D printed and embedded sensors for satellite qualification structures,' 2015 IEEE SENSORS - Proceedings, (2015):pp. 1, doi:10.1109/ICSENS.2015.7370541. 
[4] S.-Y. Wu et al., '3D-printed microelectronics for integrated circuitry and passive wireless sensors,' Microsystems \& Nanoengineering, 1(2015)(April):p. 15013, doi:10.1038/micronano.2015.13.

[5] J. Delamare et al., '3d printed biomimetic whiskerbased sensor with co-planar capacitive sensing,' in '2016 IEEE SENSORS,' 2016 pp. 1-3, doi:10.1109/ICSENS.2016.7808631.

[6] OpenScad, 'The programmers solid 3d cad modeller,' http://www . openscad.org.

[7] S. D. Senturia, Microsystem Design, vol. 49, 2001, ISBN 0792372468, doi:10.1007/0-306-47601.

[8] Stratasys, 'Connex3D printers,' http://www . stratasys.com/3d-printers/production-series/ connex3-systems.

[9] ORD, 'RoVa3D printers,' http://www.ordsolutions . com/rova3d/.

[10] Voxel8, 'Multi-Material Digital Manufacturing,' http:// www. voxel8. com.

[11] Proto-pasta, 'Conductive pla,' https://www. proto-pasta.com/pages/conductive-pla, 2016.

[12] D. Fitz-Gerald et al., 'Manufacturing and characterization of poly (lactic acid)/carbon black conductive composites for fdm feedstock: An exploratory study,' .

[13] J.-C. Huang, 'Carbon black filled conducting polymers and polymer blends,' Advances in Polymer Technology, 21(2002)(4):pp. 299, doi:10.1002/adv.10025.

[14] J. T. Muth et al., 'Embedded 3D printing of strain sensors within highly stretchable elastomers,'
Advanced Materials, 26(2014)(36):pp. 6307, doi:10.1002/adma.201400334.

[15] V. Muller et al., 'Sensor design and calibration of piezoresistive composite material,' 2015 doi:10.1109/ICSENS.2015.7370488.

[16] S.-H. Ahn et al., 'Anisotropic material properties of fused deposition modeling abs,' Rapid Prototyping Journal, 8(2002)(4):pp. 248.

[17] Z. Levin et al., 'Flexible latex - polyaniline segregated network composite coating capable of measuring large strain on epoxy,' Smart Materials and Structures, 22(2013)(1), doi:10.1088/0964-1726/22/1/015008.

[18] C. Cochrane et al., 'Design and development of a flexible strain sensor for textile structures based on a conductive polymer composite,' Sensors, 7(2007)(4):pp. 473.

[19] Flashforge, 'Creator pro 3d printer,' http://www. flashforge.com/creator-pro-3d-printer/.

[20] Flexionextruder, 'Dual flexion retrofit kit,' https:// flexionextruder. com/shop/dual/.

[21] Ninjatek, 'NinjaFlex flexible filament,' https: //ninjatek.com/products/filaments/ninjaflex/.

[22] Blackmagic3D, 'Conductive flexible tpu,' https://www . blackmagic3d.com/Conductive-p/bm3d-tpu-175. htm.

[23] seniam.org, 'Seniam website,' http://seniam.org. 